\title{
A New Method of Error Detection in SPIHT Coded Images
}

\author{
Liu Ling \\ School of Information Technology Engineering \\ Tianjin University of Technology and Education,TUTE \\ Tianjin, China 30022
}

\author{
Zhang Xinghui \\ School of Information Technology Engineering \\ Tianjin University of Technology and Education,TUTE \\ Tianjin, China 30022
}

\author{
Liang Wei \\ School of Information Technology Engineering \\ Tianjin University of Technology and Education,TUTE
}

Tianjin, China 30022

\begin{abstract}
In this paper we propose a novel approach based on pieces of information detection in order to improve the error detection efficiency and accuracy of the set partitioning in hierarchical trees (SPIHT) algorithm. The erroneous block whose parameter is greater than the threshold is dealt with error concealment according to error concealment neighbor way. The simulation results show that the algorithm is simple, and can effectively find the errors. Comparing with the method without error detection and concealment, the decoder PSNR of reconstructed image has improved 0.3-1.1dB.

Keyword-SPIHT, error detection
\end{abstract}

\section{INTRODUCTION}

When image and video are transmitted over noise channels their data are more (or less) missing or incorrect. When decoding them the error detection is a necessary step. Only if the error position is located accurately, the error concealment can be done effectively. Therefore, error detection technique plays a very important role in the decoder. At the same time the quality of the images is provided.

Set partitioning in hierarchical tree (SPIHT) [1] developed by Said and Pearlman, provides high performance in image compression with low complexity. Recently, image and video coders based on wavelet have been quite successful. Though SPIHT based coder is very efficient for image coding, it is extremely sensitive to bit errors at the same time.

Error detection methods, the existing into grammar/semantic detection and correlation detection [2-5]. Most of the existing correlation detection algorithms have high complexity, which cause delayed longer interrupt decoder. For example, Literature [6] propose a SPIHT image coding error correction solution based on partitioning bits of coding, SPIHT bits are restructuring according to each group block and then packing the data transmission together. Once appear error, it can be limited in this block, but this paper did not give an effective error detect method.

In this paper, we propose a novel approach for the detection of transmission errors. After the wavelet transform, SPIHT encoded bits are re-arranged according to spatial block representation, then the bits corresponding to a group of blocks (or slice ) are transmitted together. At the decoder, the erroneous slices are detected while decoding the image. After decoding the whole picture by measuring the inner-block correlation (IC) and interact-block correlation between block(ICB) over the adjacent blocks. These detection threshold (IC\&ICB) is updated by measuring the mean average over the error block's neighbor to locate the erroneous block. The erroneous block (whose parameter (IC\&ICB) is greater than the threshold) is dealt with error concealment according to error concealment method. This detection algorithm combined with the image correction can be effective.

\section{SPIHT IMAGE CODING}

The basic SPIHT algorithm has three passes within bit plane corresponding to three lists: LIP (insignificant pixel), LIS (insignificant set) and LSP (significant pixel). During LIP and LIS passes(also called sorting pass), for a given threshold $\mathrm{T}=2 \mathrm{~m}$, the encoder represents significance of a pixel or a set by outputting bit ' 0 ' (insignificant) or ' 1 ' (significant). Once a significant coefficient is found, its sign bit is immediately outputted. So for every significant coefficient, two bits are outputted, which will reconstruct the coefficient value at the decoder. All other bits in the sorting pass although don't reconstruct the coefficient value but refinement pass is based on these bits.

In SPIHT, the wavelet coefficients that bear the information associated with the same location and orientation are treated as a spatial-orientation tree. The SPIHT's SOT with roots in the lowest frequency sub band and out of each $2 * 2$ root nodes, one node has no descendants and every other coefficient has four offspring in the higher frequency sub band of the same orientation, thus forming trees. The set of four LL band coefficients and their descendents (if any) represent the wavelet transform of $2 n+1 * 2 n+1$ square block of the image, where $n$ is the number of decomposition levels. 


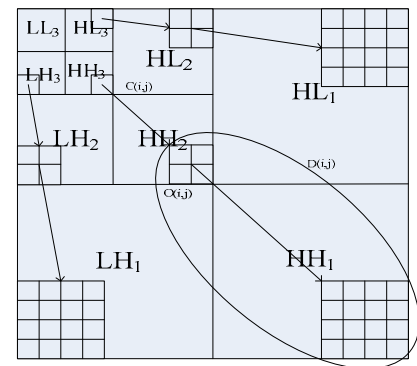

Figure 1 SPIHT's spatial orientation tree

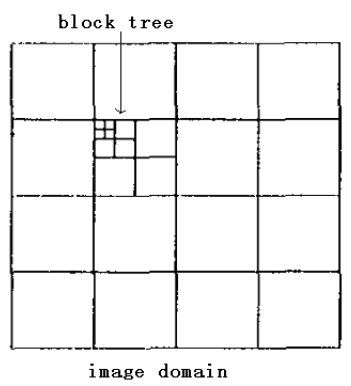

Figure 2(a) spatial orientation tree after block

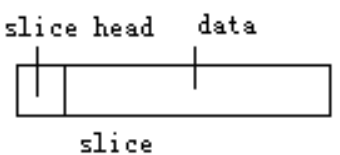

Figure 2(b) structure of slice

There are two parts in the SPIHT algorithm the sorting pass get important information and the refinement pass get refinement information. Important information uses variable length coding. Then it decodes according to the front of the scanning sequence to ensure the important position of the coefficient. Therefore, sorting bits except the sign bits as they are highly sensitive to errors causes catastrophic decoding failure. The sign and refinement bits are less sensitive and they only distort images locally without affecting the synchronization between the encoder and decoder. The refined information includes the quantification and symbols of important coefficient. Error of the refined information affects only the symbols and size of the important coefficient, and will not cause the spread of error. In this paper, we use re-arranged SPIHT bits stream and pack the re-arranged block then transmitted together. At the same time we should pack the head message. According to this encoding method we proposed two steps error detection.

\section{ERROR DETECTION}

Error bit stream causes the data inconsistency. And the data inconsistency causes error block. Error block not only lead to not smooth interface between error block and its neighborhood but also occurs the data malformation within the block. Therefore we first detect whether the data is consistent second detect the correlation.

Step 1: syntax error detection:

Because of SPIHT is volatile length coding, error condition is very complex. If bit stream are erroneous, it effects the blocks of the slice in which it is present, and at the decoder one of the following conditions may occur:

1. Decoders decode the stream, but the result overstep the normal range

2. All the stream in the stream, but the amount of block in a slice are not up to snuff.

If decoder detects any of the above conditions in a slice, it mark that slice as erroneous and continue decoding the next slice. Once the whole picture is decoded, the decoder re-visits the erroneous slice.

Step 2: correlation error detection

The normal image in a particular area and the interface between the adjacent blocks should be smooth. If we use a numerical data presented, the value of the data should be within a certain smaller range. But the data of error block is irregular, the inner-block correlation and the interact-block correlation between block is irregular. The value of then is large. So we use this correlation characteristic to do the error detection

When the position of error slice be marked we use the internal correlation for a block and external correlation between the block to do the error detection. First IC (inner-block correlation) is the internal correlation of the $\mathrm{M}$ * N block :

$$
I C(C)=\frac{\sum_{y=0}^{N-1} \sum_{x=0}^{M-1}|f(x, y)-f(x-1, y)|+\sum_{x=0}^{M-1} \sum_{y=0}^{N-1}|f(x, y)-f(x, y-1)|}{N \times(M-1)+M \times(N-1)}
$$

Formula (1), $f(x, y)$ is the pixel values of the current block located as $(x, y)$.

Then ICB (interact-block correlation between block) is the current block and their adjacent block correlation :

$$
I C B(C)=\frac{\sum_{x=1}^{N}\left|I_{t}(x, y)-I_{t}(x, y-1)\right|+\sum_{x=1}^{N}\left|I_{b}(x, y)-I_{b}(x, y+1)\right|}{(N+M)}
$$

In formula $(2), I_{t}(x, y)$ and $I_{b}(x, y)$ are respectively express the boundary pixels of current block at top and bottom. Mesure IC and ICB for each block within slice which adjacent erroneous slices. Because of the data in errror block is malformed the value of IC and ICB is larger. According to the previous message of error slice, we use formula (3) to deal with the data for further detection:

$$
I C(C)<k_{1} A
$$




$$
\operatorname{ICB}(C)<k_{2} B
$$

In the formula (3)(4) $k_{1} k_{2}$ is constant, can take intrger within (1-3). The threshold $\mathrm{A}$ and $\mathrm{B}$ calculates by the adjacent correct block of current block. We can use formula (5)(6) to calculate A, B:

$$
\begin{gathered}
A=\frac{I C_{t}(C)+I C_{b}(C)}{2} \\
B=\frac{I C B_{t}(C)+I C B_{b}(C)}{2}
\end{gathered}
$$

In formula $(5)(6) \quad I C_{t}(C) I C_{b}(C)$ express the internal correlation of top and bottom adjacent blocks. $I C B_{l}(C) I C B_{r}(C)$ express the external correlation around the current block. The principle diagram for correlation detection as follow:

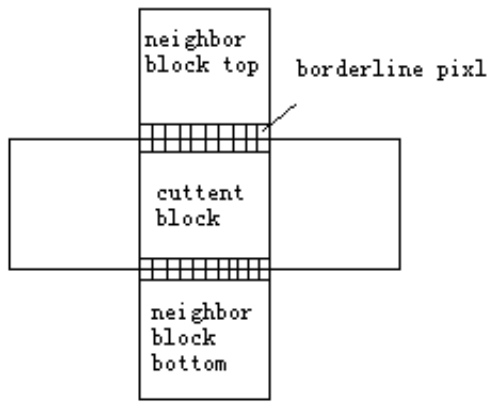

Figure 3. The block correlation detection

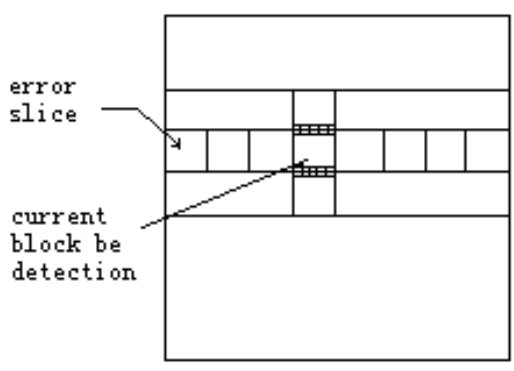

Figure 4. In the image of the block

After completing error detection, we do error concealment. Error concealment relieves the visual degradation by interpolating the erroneous or lost data at the decoder. We use simple method correction that can be built from correlated adjacent blocks of error block. Then we can reconstruct final image .

\section{Simulation Results}

In the process of simulation experiment, we simulated through a lot of error image experiment (in experimental process, we use the gray image, the size of image select 256,512 etc, code rate of 1 , error rates within scope 5-20 percentage). Through experiment we get to the optimal threshold for 3A 3B of error detection.
Usually error detection and error correction combined in practical application can receive good effect. Many correction methods have been proposed. The method we used in simulation is simple, it reconstruct the error block by correlated adjacent blocks. And the weight of the correlated adjacent blocks which calculate by their distance of the error block is inversely proportional. We adopt lena512 gray image to simulation. In lose less reconstructed image, we use four error rate $5 \%, 10 \%, 15 \%, 20 \%$. The quality of error image quality and restore image compares in table I.

Table I. the quality of lena512 grayscale image

\begin{tabular}{|l|l|l|l|}
\hline $\begin{array}{l}\text { Various error } \\
\text { rates }\end{array}$ & $\begin{array}{l}\text { Error image } \\
\text { /PSNR }\end{array}$ & $\begin{array}{l}\text { Restore image } \\
\text { /PSNR }\end{array}$ \\
\hline $5 \%$ & 36.4045 & 36.7018 & \\
\hline $10 \%$ & 36.0457 & 36.7099 & \\
\hline $15 \%$ & 35.7648 & 36.6519 & \\
\hline $20 \%$ & 35.4664 & 36.4808 & \\
\hline
\end{tabular}

Simulation results are show in the table: Table (1) shows the results of gray image peak signal-to-noise ratio (PSNR), unit of measure of decibels $(\mathrm{dB})$, and bit rate 1 . From the table we can clearly see that our error detection method is applied to improve the quality of images and especially the error rate is high that the quality of the image has improved significantly.

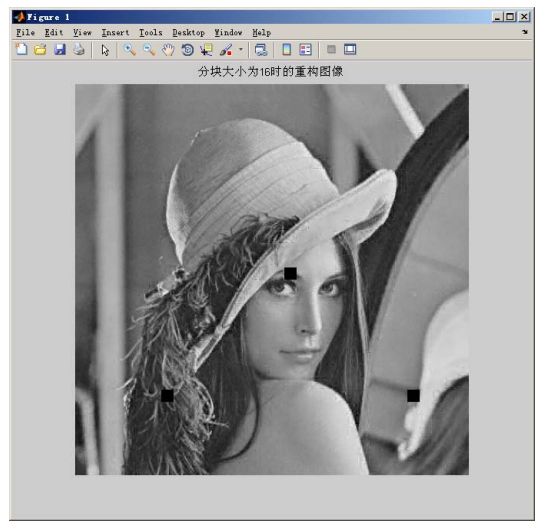

Figure 5 (a) error image of 5\% error rate

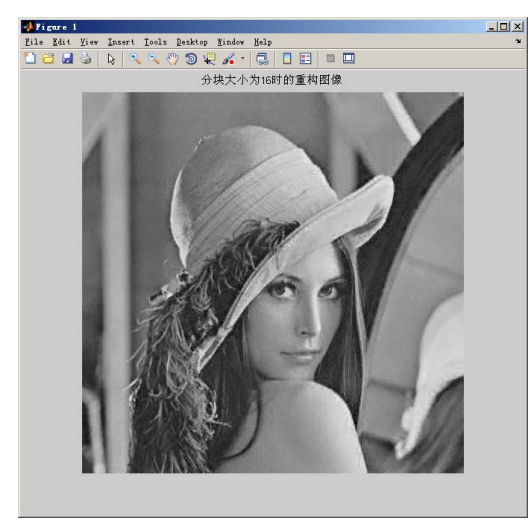

Figure 5 (b) restoration image of 5\% error rate 
Figure 5(a) has 5\% error rate, but figure 5(b) is the image after error detection and concealment (image restoration) is applied.

\section{CONCLUSIONS}

With existing image error recovery technology as the foundation, focusing on SPIHT decoding error detection technology, aiming at the characteristics of streaming SPIHT and decoder of real-time require, we propose SPIHT based two-step error streaming method. This kind of coding method is done by re-arranging bits of block, packetizing and then transmitting each slice independently without sacrificing the compression efficiency. At the decoder the erroneous MT within a erroneous slice is detected by measuring the inner-block correlation (IC) and interact-block correlation between blocks (ICB) over the neighboring blocks. The simulation results show that the method combined with the error correction effectively eliminate the error, so as to enhance the quality of reconstructed decoding.

\section{REFERENCES}

[1] Said, W. A. Pearlman. A new, fast, and efficient image codec based on set partitioning in hierarchical trees[J]. IEEE Trans. Circuits and Systems for Video Technology.1996,6(3): 243-250.

[2] Wen JC, Jin JL. Detection and concealmentof transmission errors in H. 261 Images[ J]. IEEE Transactions on Circuits and Systems for VideoTechnology, 1998,8(1): 74 84.

[3] WangY, Zhu Q F. Error control and concealment for video communication: a review $[\mathrm{J}]$. Proceedings of the IEEE, 1998,86(5):974 997.

[4] Ekram Khan, Stefan Lehmann, HiroshiGunj,iet al. Iterative error detection and correction of H. 263 coded video forwireless networks[J]. IEEE Transactions on Circuits and Systems for Video Technology, 2004,12(14): 1294 1306.

[5] Yang Wen-yong, Huang Chao, Li Jin-tao, Xiao Fang-ming Detection and Concealment of Video Errors for AVS Decoder. Joumal of Image and Graphics,2008,(11),13:2106-2111

[6] Ekram Khan and Mohammed Ghanbari.Error detection and Correction of Transmission Errors in SPIHT Coded Images[A].IEEE ICIP 2002:689-692 